\title{
Investigation of local heat transfer enhancement in generic liquid metal-cooled fuel assemblies with empirical models
}

\author{
W. Jaeger ${ }^{1}$ and W. Hering ${ }^{2}$ \\ ${ }^{1}$ Karlsruhe Institute of Technology, Institute of Fusion and Reactor Technology, \\ Kaiserstraße 12, 76131 Karlsruhe, Germany, \\ ${ }^{2}$ Karlsruhe Institute of Technology, Institute for Neutron Physics and Reactor Technology, \\ Hermann-von-Helmholtz-Platz 1, 76344 Eggenstein-Leopoldshafen, Germany, \\ wadim.jaeger@kit.edu, wolfgang.hering@kit.edu,
}

\begin{abstract}
The heat transfer in liquid metal-cooled rod bundles is modeled with a knowledgebased best-estimate system code. Thereby, the focus is on the heat transfer enhancement due to flow perturbations. These perturbations are caused by local geometrical variations, such as sudden expansions and contractions, in the flow channel. The accurate calculation of the heat transfer is important for the safety demonstration of, e.g., subassemblies. Safety related parameters, such as fluid and wall temperature, have to satisfy certain limits during normal and off-normal operation as well as during accidents. Up to now, fully developed flow is assumed for heat transfer in liquid metal-cooled rod bundles. The effects of local heat transfer enhancements are ignored in best estimate-system codes. The currently used empirical heat transfer models are functions of the Péclet number only. Several experimental and numerical investigations show that flow perturbations induce higher heat transfer due to increased turbulences, accelerated flows and secondary motions. In this paper, the effects of the entrance region and the presence of spacer grids on the heat transfer are investigated. Empirical models for that are selected and applied. These empirical models are functions of the Péclet number, the geometrical perturbation and the distance from the perturbation in flowing direction. The calculated heat transfer coefficients at the bundle entrance and in the vicinity of spacer grids are twice as high compared to bare rod bundles under fully developed flow condition without any flow perturbation. Due to the higher heat transfer, lower wall temperatures are to be expected. This provides additional safety margins during normal and off-normal operation as well as during accidents. Furthermore, the considerable increase of heat transfer shows that existing perturbations have to be considered to obtain accurate and reliable results.
\end{abstract}

\section{Introduction}

The heat transfer to a generic liquid metal-cooled rod bundle is investigated. The geometric variations inside the rod bundle causing flow perturbations are thereby of special interest. These are the entrance to a rod bundle and the spacer grids along the rod bundle. In both cases, the geometric variations, basically sudden reductions of the flow area, will influence the heat transfer 
in a positive way. On the one side, the flow area reduction will accelerate the fluid resulting in higher Reynolds numbers and therefore higher Nusselt numbers. On the other side, spacer grids introduce additional turbulences and secondary flows, resulting in improved heat removal from hot surfaces. Taking these effects into account during heat transfer calculation, higher values for the heat transfer coefficient will be obtained. In the following Figure 1, the relative Nusselt numbers are plotted. On the left side the relative Nusselt numbers for the spacer grid region (labelled RNS) are shown [1], while on the right side the relative Nusselt numbers for the entrance region (labelled RNE) of a flow channel are shown [2]. The relative Nusselt numbers are thereby the ratio of the local Nusselt number over the developed Nusselt number. The local Nusselt number is the Nusselt number as a function of the position downstream of the flow perturbation. To normalize over all bundle geometries, the RNSs are plotted as functions of $l / d$. This is the dimensionless distance from the spacer grid in downstream direction. Thereby, $l$ is the distance and $d$ is the hydraulic diameter of the rod bundle. For example, a value of $5 \mathrm{l} / \mathrm{d}$ indicates a position 5 hydraulic diameters downstream of the spacer grid. On the right side of Figure 1 the RNEs are shown as a functions of the distance from the entrance. To avoid confusion with the distance from the spacer grid $(l)$, the RNEs are plotted as function of $x / d$ ( $x$ is the distance from the entrance and $d$ is the hydraulic diameter). The developed Nusselt number is the Nusselt number, which will be obtained if no geometrical variations and no flow perturbations are considered. It is the Nusselt number of an infinite long and bare rod bundle. This Nusselt number is labelled $\mathrm{Nu}_{\infty}$.

In both plots the relative Nusselt number is greater than unity. In the vicinity of spacer grids and at the entrance of the rod bundles higher Nusselt numbers are present. Therefore, higher heat transfer coefficients are present, too. In both cases, the local Nusselt number is almost twice as high as the developed Nusselt number. It is also visible that the effect of higher heat transfer is not limited to the exact position of the flow perturbation. In a decaying trend, higher heat transfer is present till 20 hydraulic diameters downstream of the flow perturbation. Because several spacer grids are installed along the rod bundle, the local heat transfer enhancement takes place several times in a rod bundle. Based on the distance between spacer grids, it is possible that at no time and at no location along the rod bundle developed flow will be established.
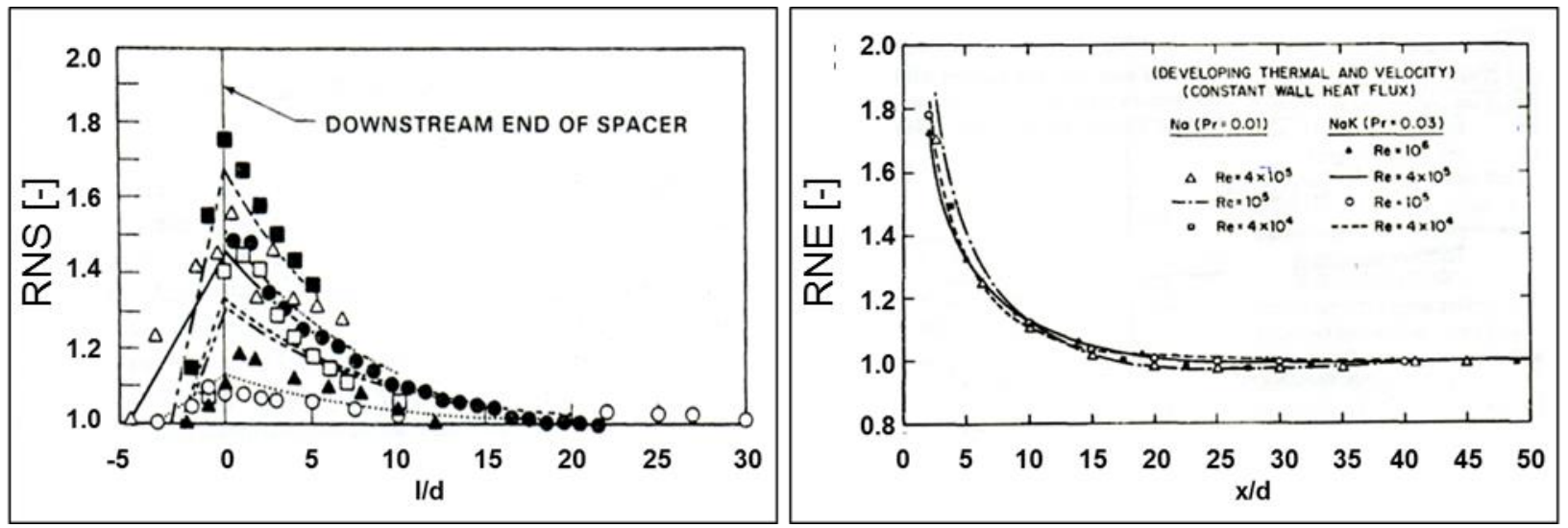

Figure 1 Heat transfer enhancement in the vicinity of a spacer grid (left side) [1] and heat transfer enhancement at the entrance of a flow channel (right side) [2] 
In order to calculate, say, the wall temperature of the rods in an accurate way, it is necessary to consider these effects on the heat transfer process. Including these effects will help to reduce conservatism in the design of rod bundles. Due to higher heat transfer coefficients lower temperatures are to be expected assuming identical boundary conditions (e.g., mass flow rate, heat flux, material and fluid properties, etc.).

To account for these heat transfer enhancements, empirical models are used. Dedicated models for the heat transfer in the vicinity of spacer grids and at the entrance are available in the best estimate system code TRACE. TRACE follows a 2-Fluid, 6-Equation approach for the conservation of mass, momentum and energy for the liquid and vapour phase of the fluid. In the present case, only the liquid phase is considered. To close the conservation equations, empirical closure laws are needed. Here, only the wall-to-fluid heat transfer is needed. [3] The calculation procedure for the heat transfer to a liquid metal-cooled infinite bare rod bundle is the following:

$$
\begin{gathered}
q_{\text {wall-to-liquid }}=h \cdot\left(T_{\text {wall }}-T_{\text {liquid }}\right) \\
h=\mathrm{Nu}_{\infty} \cdot \frac{k}{d_{\text {hydraulic }}} \\
\mathrm{Nu}_{\infty}=7.55 \cdot\left(\frac{p}{d}\right)-20.0 \cdot\left(\frac{p}{d}\right)^{-13}+\frac{3.67}{90}\left(\frac{p}{d}\right)^{-2} \cdot(\mathrm{Pe})^{0.56+0.19 \cdot\left(\frac{p}{d}\right)}
\end{gathered}
$$

The Nusselt number correlation above is taken from Ushakov et al. [4]. This correlation can be used for Péclet (Pe) numbers below 4000 and pitch-to-diameter ratio (p/d) between 1.2 and 2.0. The applicability of this correlation for liquid metal rod bundle flow was validated in [5].

To account for the entrance region and the spacer grid, Eq. (3) has been extended to:

$$
\mathrm{Nu}_{\text {bundle }}=\mathrm{Nu}_{\infty} \cdot \mathrm{RNS} \cdot \mathrm{RNE}
$$

The presented approach is identical for other system codes like RELAP5, CATHARE, ATHLET, MARS, etc. and can therefore be adopted one-to-one. TRACE serves only as a demonstrator for the presented Nusselt correlations. Therefore, Eq. (4) has been implemented into the TRACE source code by the authors.

A generic rod bundle is proposed based on a literature survey on the design of rod bundles for liquid metal-cooled systems and reactor concepts. The heat transfer is calculated with the abovementioned Nusselt number correlation. The independent variables of this study are the Péclet number, the rod diameter, the pitch-to-diameter ratio and the dimensionless distance from the flow perturbation. The calculation of RNS, RNE and the generic rod bundle design are given below. 


\section{Heat transfer enhancement by spacer grids}

Spacer grids are a common way, besides wire wrappers, to keep the pins in their intended position inside the rod bundle. Several liquid metal cooled reactor concepts and related experimental facilities have been foreseen to use spacer grids (CIRCE-ICE, EFIT, ELSY, HELIOS, LCTF, PDSXADS, XT-ADS, THESYS, ...). Therefore, it is necessary to investigate their influence on the thermal-hydraulics of the overall rod bundle. The idea behind the heat transfer enhancement due to spacer grids is based on an analogy of momentum and heat transfer. Thereby, it is irrelevant what kind of fluid is flowing through the spacer grid. This model is applicable to fluids with Prandtl numbers close to unity, like water or gases, as well as to fluids with very low Prandtl numbers like liquid metals. The theory states that the difference between the pressure drop $(\Delta p)$ in a bare rod bundle and a rod bundle with spacer grids is identical to the difference between the heat transfer of a bare rod bundle and a rod bundle with spacers $[1,6]$. The local pressure drop in a flow channel is calculated according to:

$$
\Delta p_{\text {local }}=\frac{1}{2} \cdot \sum\left[\rho \cdot v^{2} \cdot\left(f \cdot \frac{l}{d}+K\right)\right]
$$

In the absence of a spacer grid the form loss coefficient $K$ is zero, while in the presence of a spacer the form loss coefficient $K$ is larger than zero. The following ratio can be considered:

$$
\frac{\Delta p_{\text {spacer }}}{\Delta p_{\text {no spacer }}}=\frac{\frac{1}{2} \cdot\left[\rho \cdot v^{2} \cdot\left(f \cdot \frac{l}{d}+K_{\text {grid }}\right)\right]}{\frac{1}{2} \cdot\left[\rho \cdot v^{2} \cdot\left(f \cdot \frac{l}{d}\right)\right]}=\frac{f+K_{\text {grid }} \cdot \frac{d}{l}}{f}=1+K_{\text {grid }} \cdot \frac{d}{f \cdot l}
$$

According to Rehme [7], the form loss coefficient is based on a Reynolds dependent term and a flow area reduction dependent term. Thereby, the actual design of the spacer grid is of no importance (honeycomb design, triangular design, etc.). The blockage ratio $(\varepsilon)$ is the important parameter. Considering the following for the form loss coefficient

$$
K_{\text {grid }}=K_{\text {Reynolds }} \cdot \varepsilon^{2}
$$

yields:

$$
\frac{\Delta p_{\text {spacer }}}{\Delta p_{\text {no spacer }}}=1+K_{\text {Reynolds }} \cdot \varepsilon^{2} \cdot \frac{d}{f \cdot l}=1+\text { Const } \cdot \varepsilon^{2} \text {. }
$$

With

$$
\frac{\Delta p_{\text {spacer }}}{\Delta p_{\text {no spacer }}}=\frac{\mathrm{Nu}_{\text {spacer }}}{\mathrm{Nu}_{\text {no spacer }}}
$$

the following relationship can be obtained: 


$$
\frac{\mathrm{Nu}_{\text {spacer }}}{\mathrm{Nu}_{\text {no spacer }}}=\frac{\mathrm{Nu}_{\infty} \cdot \mathrm{RNS}}{\mathrm{Nu}_{\infty}}=\mathrm{RNS}=1+\text { Const } \cdot \varepsilon^{2}=1+\mathrm{A} \cdot \varepsilon^{2} \text {. }
$$

It can be seen that RNS is not like a real Nusselt number. It is not the ratio of convective and conductive heat transfer. RNS is more like a multiplier, which is unity at a sufficient distance from the flow perturbation (= developed flow). The value for the constant $A$ was estimated by Marek and Rehme [6] to be 5.55. Yao et al. [1] extended the model, because the heat transfer enhancement is not only limited to the exact position of the spacer grid, but is also present several hydraulic diameters downstream with a decaying trend. Eq. (10) is rewritten to:

$$
\frac{\mathrm{Nu}_{\text {spacer }}}{\mathrm{Nu}_{\text {no spacer }}}=\frac{\mathrm{Nu}_{\infty} \cdot \mathrm{RNS}}{\mathrm{Nu}_{\infty}}=\mathrm{RNS}=1+\mathrm{A} \cdot \varepsilon^{2} \cdot e^{-\mathrm{B} \cdot \frac{l}{d}}
$$

Constant B was originally estimated to be 0.13 . Hence, constant A now represents the magnitude, while constant B represents the decaying trend of the heat transfer enhancement. In further studies, Yao et al. [1] also addressed the effect of the mixing vanes on the flow. In addition, the behaviour during laminar and transitional flow was investigated. In the present case, only the spacer grid itself without vanes is considered. Mixing vanes are usually used for light water reactor fuel assemblies, while in liquid metal-cooled system vane-less designs are common. Furthermore, only forced turbulent flow is considered. A complete description of the heat transfer enhancement is given by Jaeger and Sánchez [8].

The presented model has been implemented by the US NRC in the system code TRACE. The applicability of this procedure was validated by Jaeger and Sánchez [8]. An example on how the Nusselt number is influenced by the spacer grid is shown in Figure 2. The magnitude and the decaying trend are represented very good from a qualitative and quantitative point of view. The main difference is the shift of the maximal values. Due to the numerical scheme, the calculated heat transfer coefficient has its maximum directly at the spacer grid inlet.

The experiments show maximal RNS values at 1.5 to 2.0 hydraulic diameters downstream of the spacer grid. The hydraulic diameter was in the range of $16 \mathrm{~mm}$. Hence, the deviations are in the range of $30 \mathrm{~mm}$ or less. With respect to the intention of system codes in general, such small deviations can be ignored. 


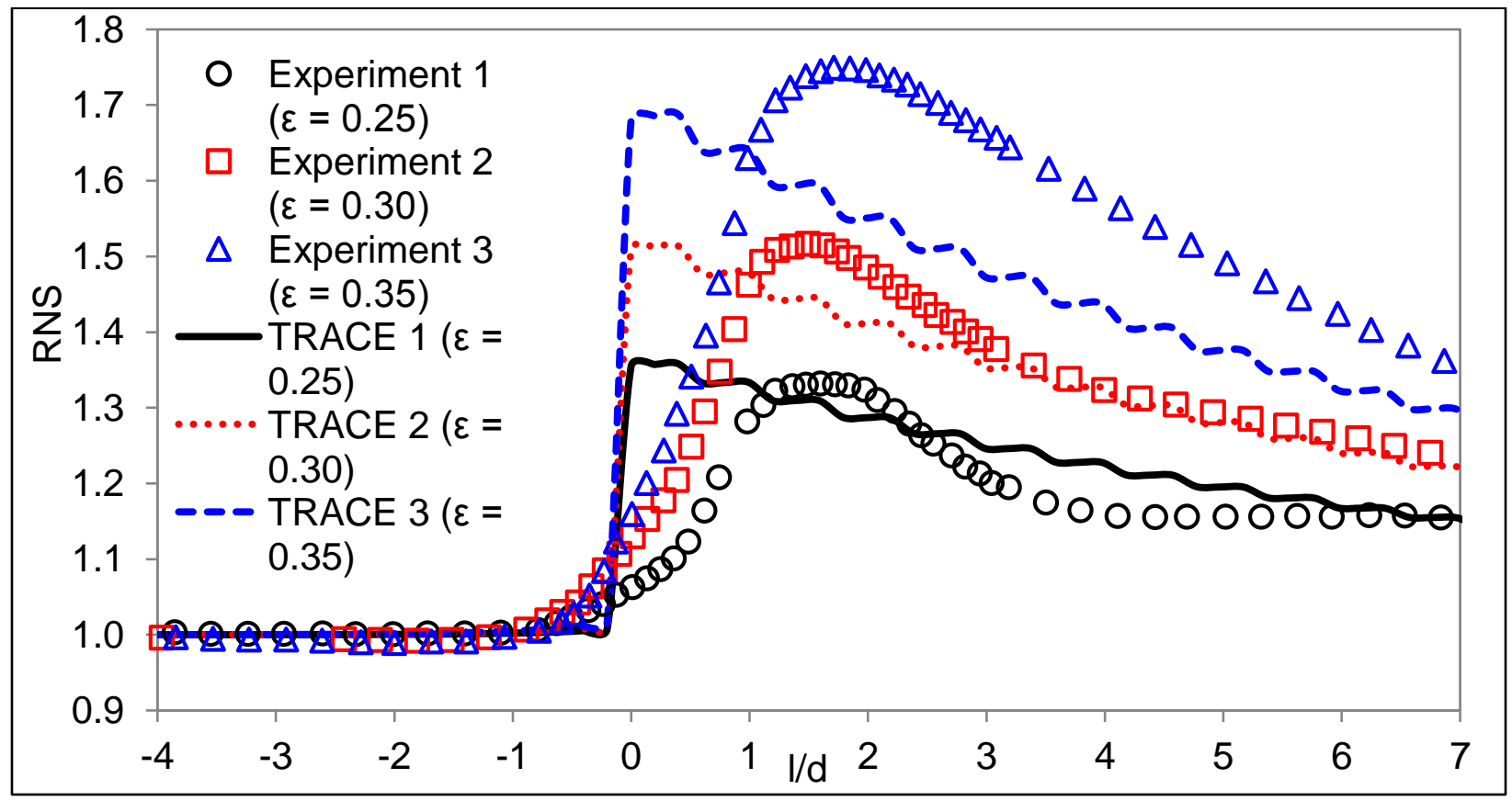

Figure 2 RNS as a function of the dimensionless distance from the spacer grid for different blockage ratios (Figure adopted from [8]. Experimental data taken from [6])

\section{Heat Transfer Enhancement at the Entrance}

The heat transfer enhancement in the entrance region of flow channels is subject to many studies. In Chen and Chiou [2] an overview on this subject is given with references to experimental and numerical investigations. Unfortunately, this paper focuses more on pipes. Hence, empirical Nusselt correlations, taking into account the heat transfer enhancement, are provided for pipe flow conditions only. A dedicated review of experiments with liquid metal-cooled rod bundles was performed by the present author [9]. The outcome of this investigation is a Nusselt number correlation accounting for the peculiarities of rod bundles. Based on five experiments, RNE, the multiplier for the entrance length effect, reads as follows:

$$
\mathrm{RNE}=1+\left(16.0-7.8571 \cdot \frac{p}{d}\right) \cdot e^{-0.135 \cdot \frac{x}{d}}
$$

This multiplier is dependent on the pitch-to-diameter ratio and the dimensionless distance from the entrance. Figure 3 shows the local Nusselt number $\left(\mathrm{Nu}_{\infty} \cdot \mathrm{RNE}\right)$ as a function of the dimensionless distance from the entrance. For the experiment shown in Figure 3 the pitch-todiameter ratio is 1.75. Three different Péclet number are considered. Even though a Péclet number of 5000 is out of range according to the range of validity of $\mathrm{Nu}_{\infty}$, Eq. (3), very satisfying results are obtained. Magnitude as well as decaying trend are represented almost perfectly for system code requirements. To account for un-physically high values due to the numerical modelling, Eq. (12) will be used for $x$ values of 4 and higher. Furthermore, no experimental data are available exactly at the entrance. All measurements have been taken at least 2 or 3 hydraulic diameters downstream of the entrance. 


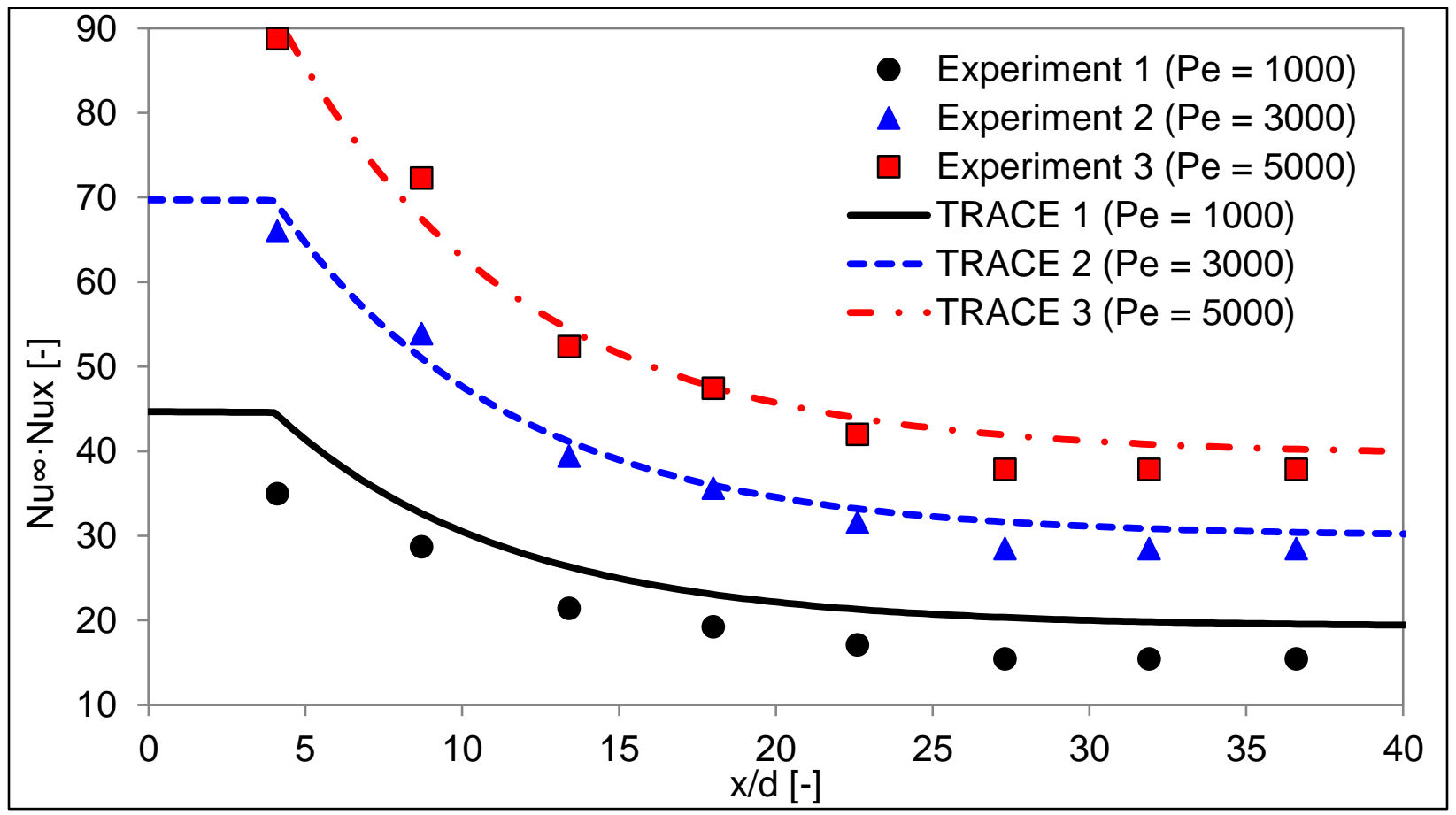

Figure $3 \mathrm{Nu}_{\infty} \cdot \mathrm{RNE}$ as a function of the dimensionless distance from the entrance for different Péclet numbers (Figure adopted from [9]. Experimental data taken from [10])

\section{Generic rod bundle}

A generic rod bundle will be designed. The knowledge of the designs of actual rod bundles is therefore necessary. A brief survey of liquid metal-cooled rod bundles revealed the following situation. Most rods bundles are in the range of 1.8 to $2.0 \mathrm{~m}$, while the active length is between $0.6 \mathrm{~m}$ and $1.0 \mathrm{~m}$. A spacer grid is installed every 0.3 to $0.5 \mathrm{~m}$. The flow area reduction due to the spacer grid is in the range of 0.4 . This is the $\varepsilon$ coefficient, depicted in Eqs. $(7,8,10,11)$. The pin diameters are between 5 and $25 \mathrm{~mm}$, while the pitch-to-diameter ratio is in the range of 1.1 to 2.0. Figure 4 shows the pitch-to-diameter ratio versus the pin diameter for liquid metal-cooled rod bundles. Two distinct areas are visible. The area with the light red background is more related to rod bundles considered for reactor applications. The light blue area is more related to fundamental heat transfer studies. For real reactor applications the design of the rod bundle does not only follow thermal-hydraulic constrains, but also neutron-physical constrains. Experiments are focusing on the fundamental phenomena and are therefore not always in relation with real application. Therefore, the light blue area in Figure 4 will be ignored for the sake of this study. 


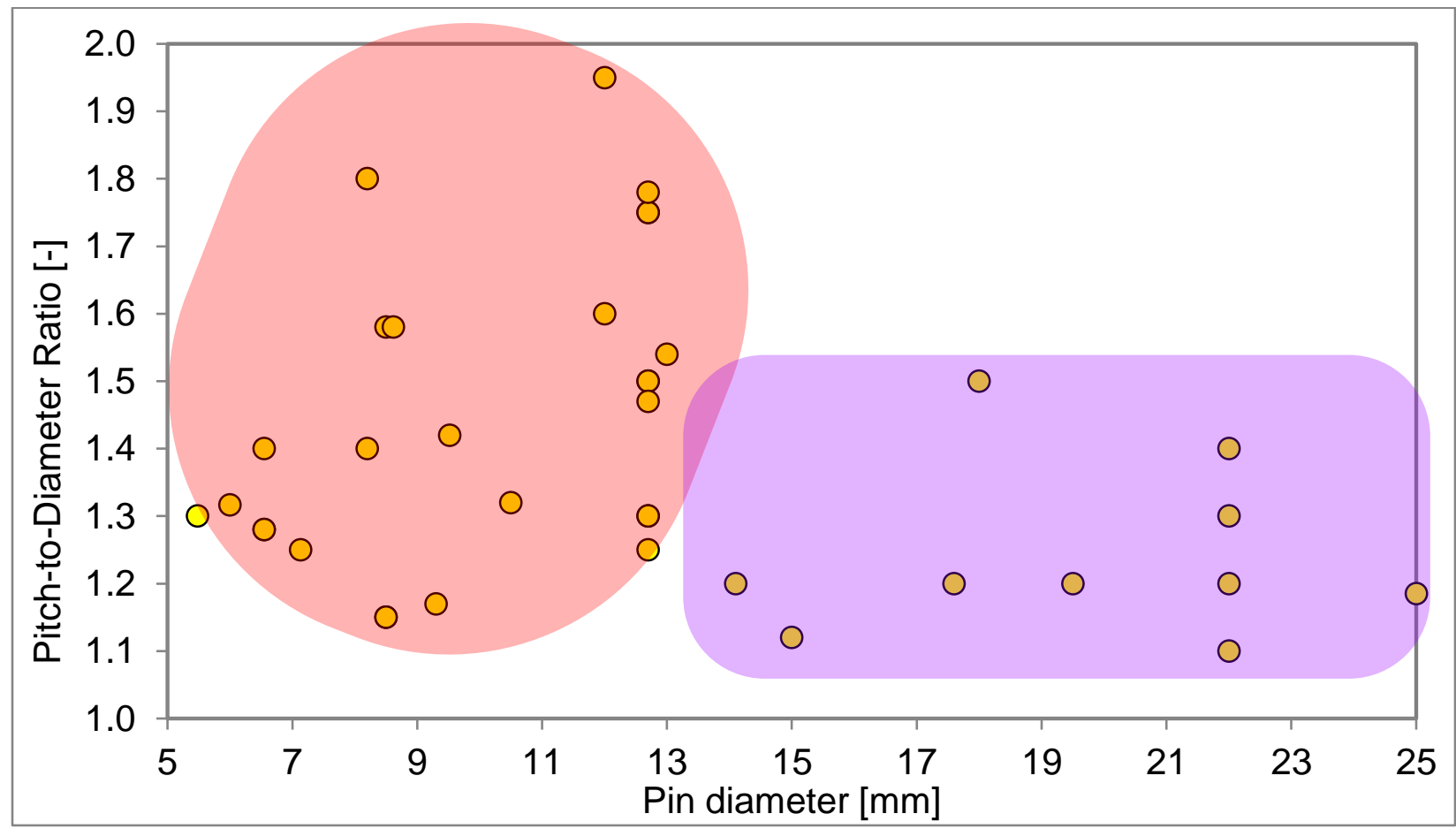

Figure 4 Pitch-to-diameter ratio versus pin diameter for liquid metal-cooled rod bundles

The number of rods per assembly is very different. In the experiments, rod numbers of 7, 19 and 36 are very common. For real plant applications or experiments with mock-up bundles of real plant assemblies, rod numbers range from 36 to more than 400. Due to the structure of system codes like TRACE, the number of rods is not of importance for the assessment of the heat transfer capabilities. The used Nusselt number correlation is an approximation on the heat transfer performance inside the bundle. Unlike sub channel codes, the rod bundle is not divided into edge, corner and center channels. Hence, the influence of the near shroud or wrapper wall is not considered. Therefore, the generic rod bundle is a bundle of infinite number of rods.

For the following study, the length of the rod bundle is fixed to $1.8 \mathrm{~m}$. The influence of the heated length will be addressed in the next section. Along the $1.8 \mathrm{~m}$ long bundle four spacer grids are installed every $0.4 \mathrm{~m}$. The blockage ratio $\varepsilon$ is fixed to 0.4 . The pin diameters are: $6,7,8,9,10,11$ and $12 \mathrm{~mm}$. The pitch-to-diameter ratios are: 1.10, 1.20, 1.30, 1.40, 1.50, 1.60 and 1.70. Hence, 49 combinations of pin diameter and pitch-to-diameter ratio are analysed.

\section{Result}

The generic rod bundle described above is modelled with TRACE. The length of $1.8 \mathrm{~m}$ is divided into 540 equidistant nodes. Such a fine nodalization was chosen to calculate, especially, the magnitude of the heat transfer enhancement. The relative Nusselt number is shown in Figure 5 as an example on how the different flow perturbations influence the heat transfer. 


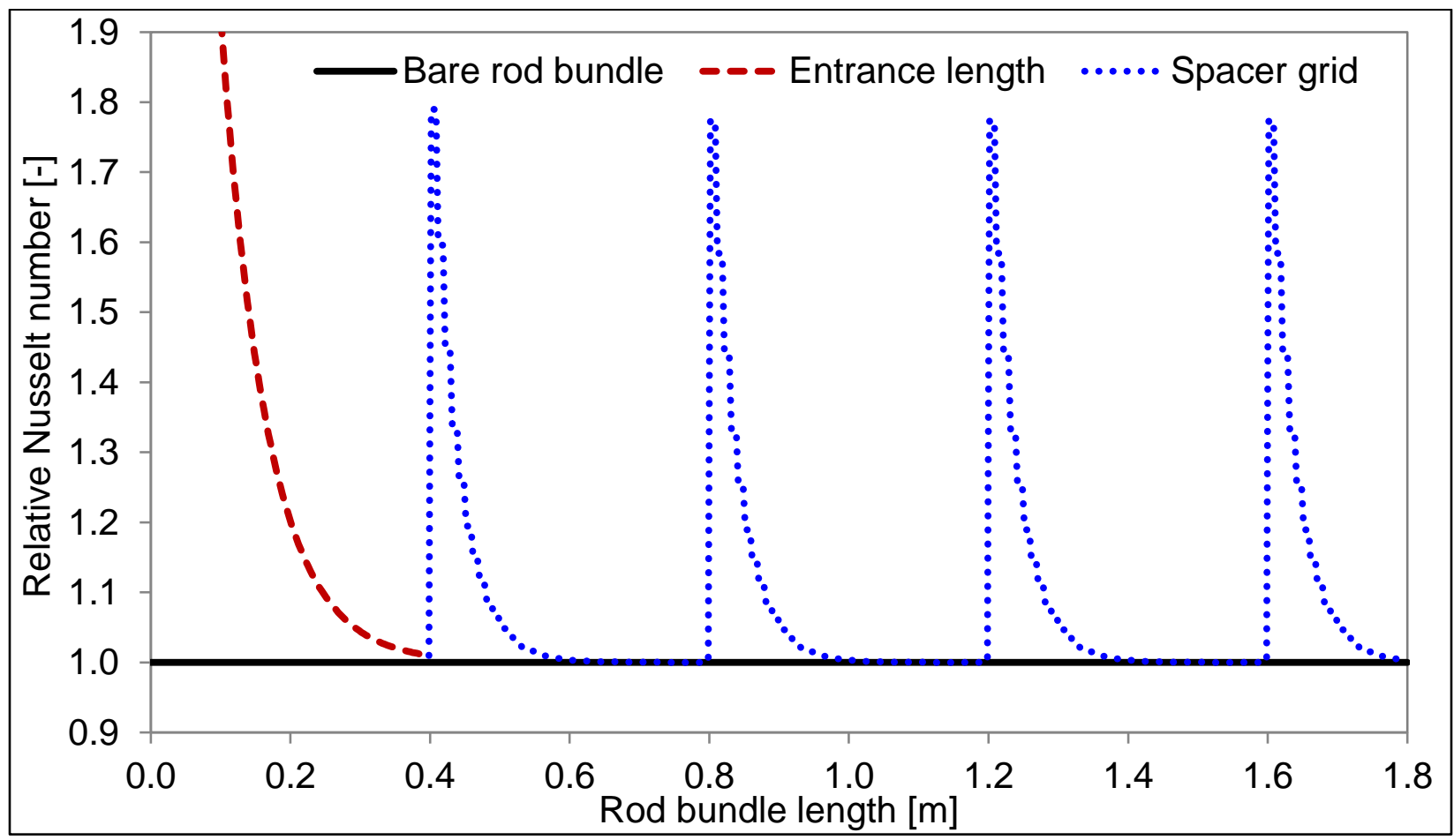

Figure 5 Relative Nusselt number versus rod bundle length indicating the impact of the entrance length and the spacer grid

The black solid line shows the relative Nusselt number for a bare rod bundle as a function of the bundle length. Because it is normalized to itself, the relative Nusselt number is unity. The red dashed line represents the influence of the entrance length, while the blue dotted line represents the influence of the spacer grids.

As mentioned above, the active length of the rod bundle is much shorter than the actual rod bundle length. Assume an active length of $1.0 \mathrm{~m}$. This leaves $0.8 \mathrm{~m}$ for the inlet and outlet section. Hence, in the active zone, up to three spacer grids could be present. The results are shown for rod bundles with $6,8,10$ and $12 \mathrm{~mm}$ pin diameters and for pitch-to diameter ratios of 1.10, 1.30, 1.50 and 1.70 in Figure 6 till Figure 9.

The figures show the distinct influence of the flow perturbation of the entrance and the spacer grid. For the entrance, the heat transfer enhancement is at least $100 \%$, meaning that the Nusselt numbers are doubled. For the spacer grids, a 70 to $90 \%$ enhancement is visible. It is also visible that larger pin diameters and pitch-to-diameter ratios increase the length of the heat transfer enhancement. Larger bundles mean larger hydraulic diameters and hence, the enhancement is visible over a longer section of the rod bundle. Due to the extension of the heat transfer enhancement zones in the wake of spacer grids, it is possible that the flow is still undeveloped before it reaches the next spacer. This is also visible for the entrance region and the first spacer grid. Especially in Figure 9 this effect is pronounced. 


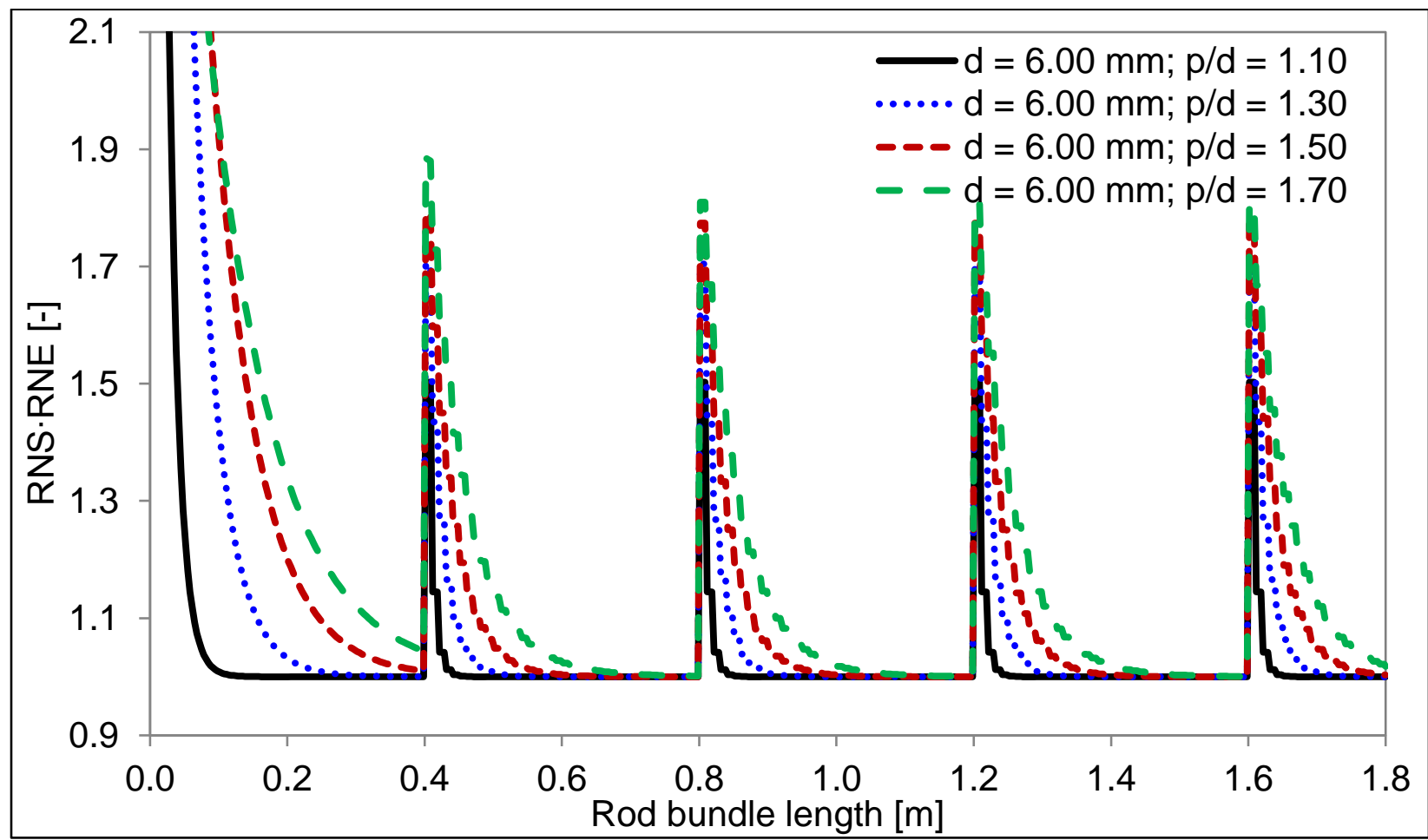

Figure 6 Relative Nusselt number (RNS·RNE) as a function of the length for a rod bundle with a pin diameter of $6 \mathrm{~mm}$

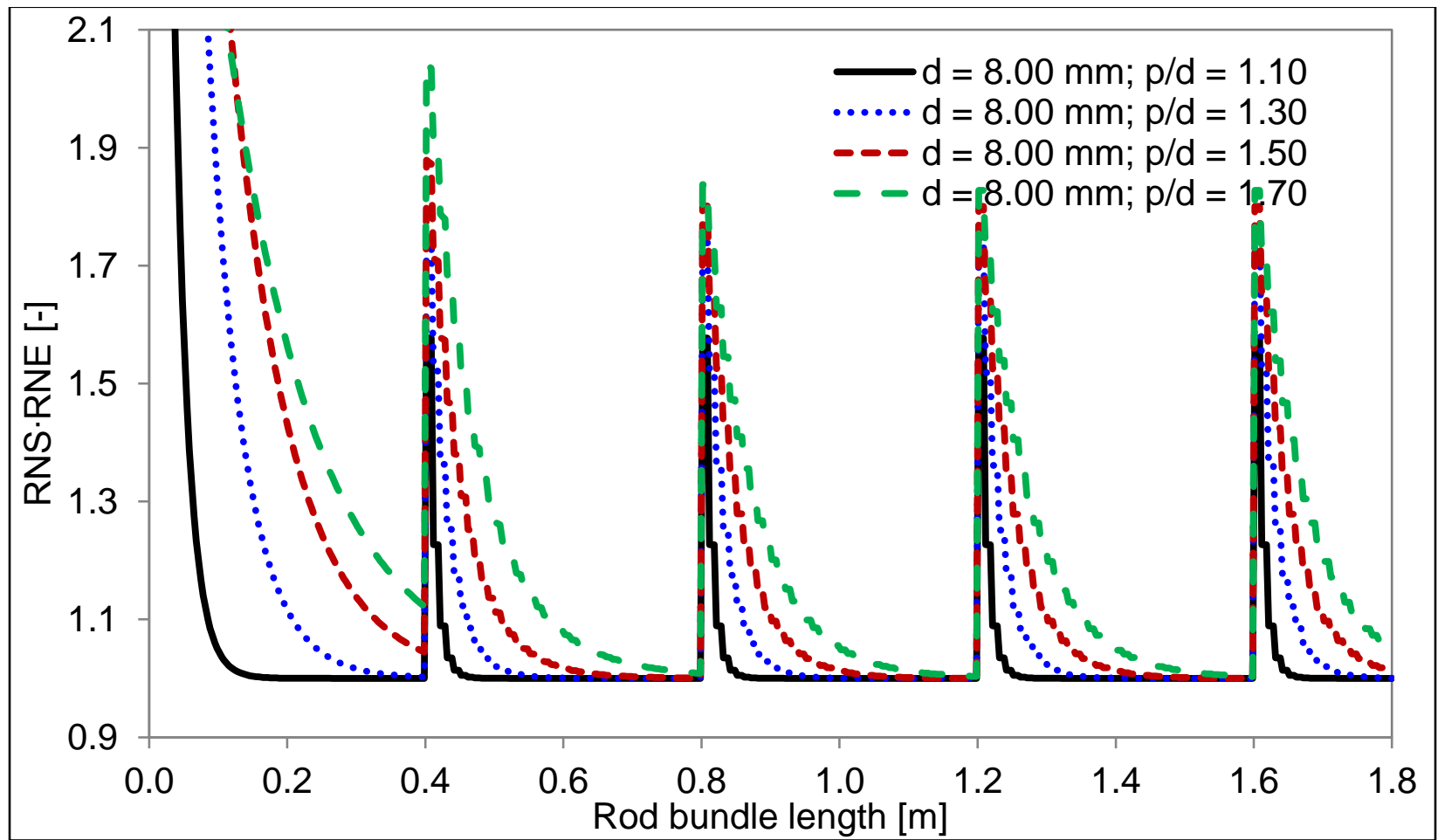

Figure 7 Relative Nusselt number (RNS·RNE) as a function of the length for a rod bundle with a pin diameter of $8 \mathrm{~mm}$ 


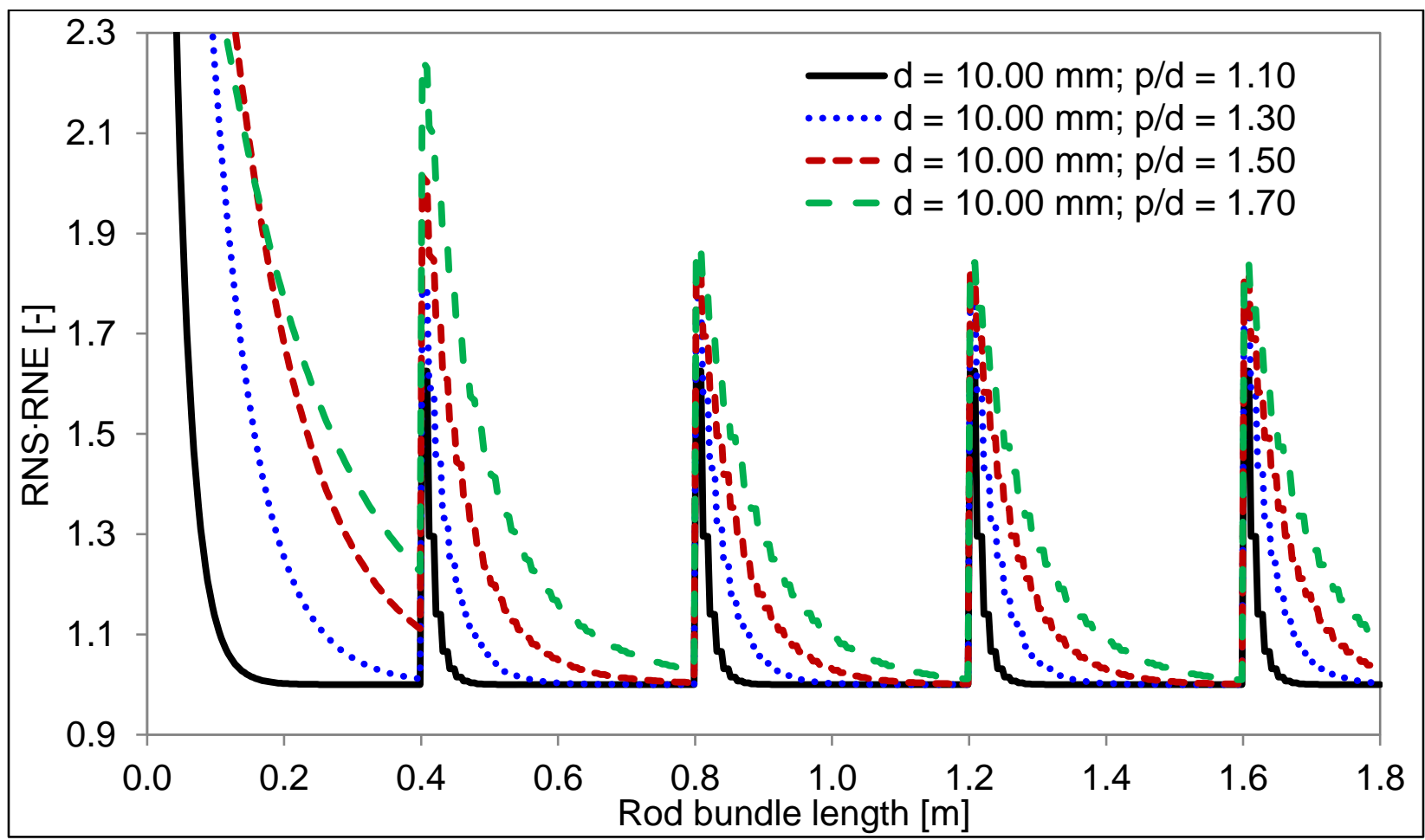

Figure 8 Relative Nusselt number (RNS·RNE) as a function of the length for a rod bundle with a pin diameter of $10 \mathrm{~mm}$

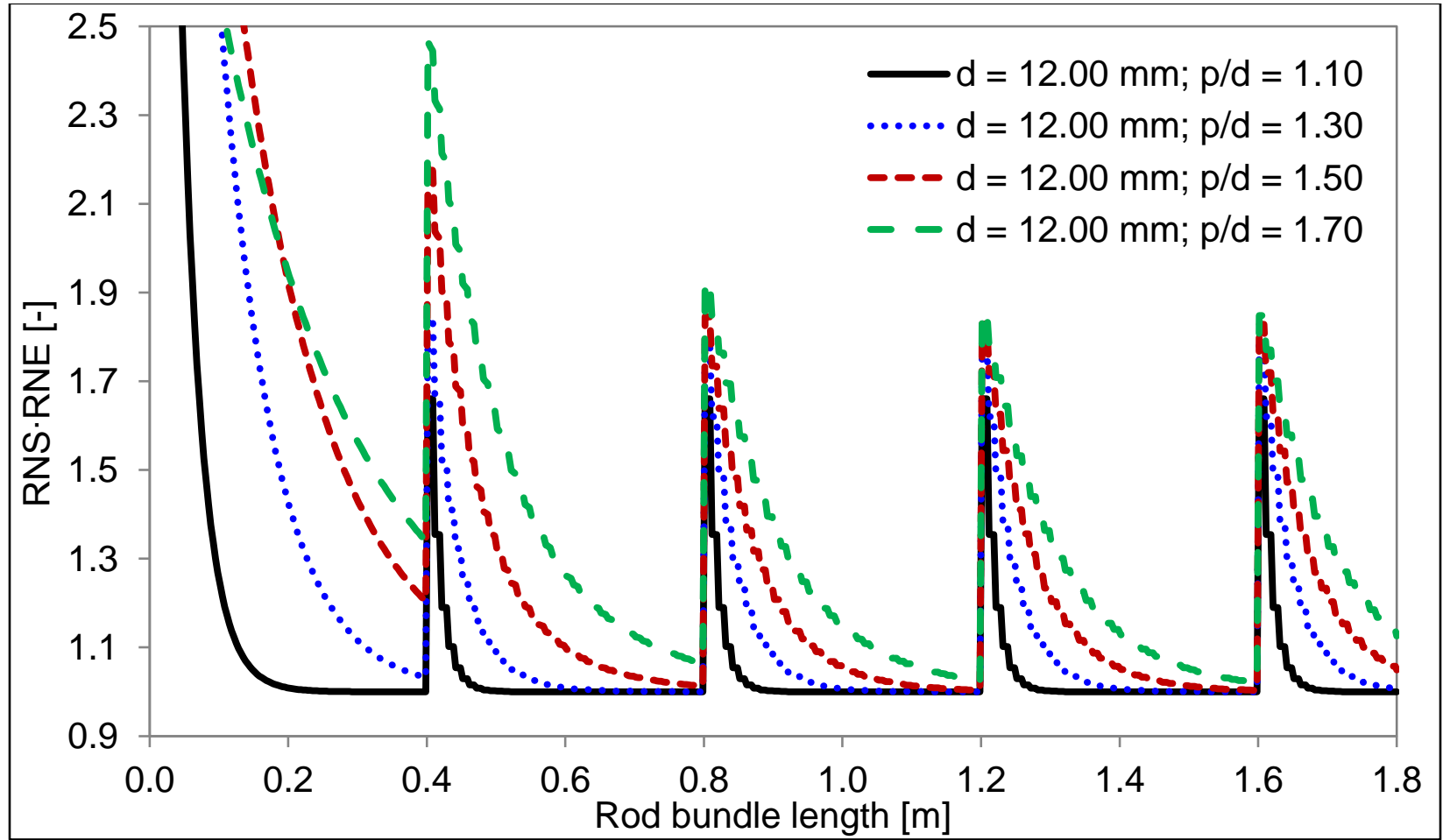

Figure 9 Relative Nusselt number (RNS RNE) as a function of the length for a rod bundle with a pin diameter of $12 \mathrm{~mm}$ 
By means of Eq. (4) it is possible to calculate the Nusselt number as a function of, among others, the rod bundle length. It is also possible to calculate the average heat transfer enhancement in the rod bundle. Therefore, the local relative Nusselt numbers have been integrated over the 540 nodes. The results are shown in Figure 10. The average RNE·RNS values are plotted as a function of the pitch-to-diameter ratio $(1.1 \ldots 1.7)$ and for different pin diameters $(6 \ldots 12 \mathrm{~mm})$. Taking a rod bundle with pin diameters of $6 \mathrm{~mm}$ and a pitch-to-diameter ratio of 1.1 will yield a 1.08 times higher heat transfer integrated over the whole bundle if a bare rod bundle is taken as reference (reference value $=1.0$ ). For a rod bundle with pin diameters of $12 \mathrm{~mm}$ and a pitch-to-diameter ratio of 1.7 the heat transfer enhancement will be 1.47 times higher as for the bare rod bundle.

\section{Discussion}

The presented study can be used as a blue print for related studies with other system codes. The correlations provided are based on a solid validation foundation. The implementation into the source code of a system code is a simple task. Therefore, it is expected that the presented result can easily be recalculated with other system codes. With the newly formulated Nusselt number correlation for liquid metal-cooled rod bundles with spacer grids, an estimation of the expected heat transfer can be performed. This is helpful during the design process of such rod bundles.

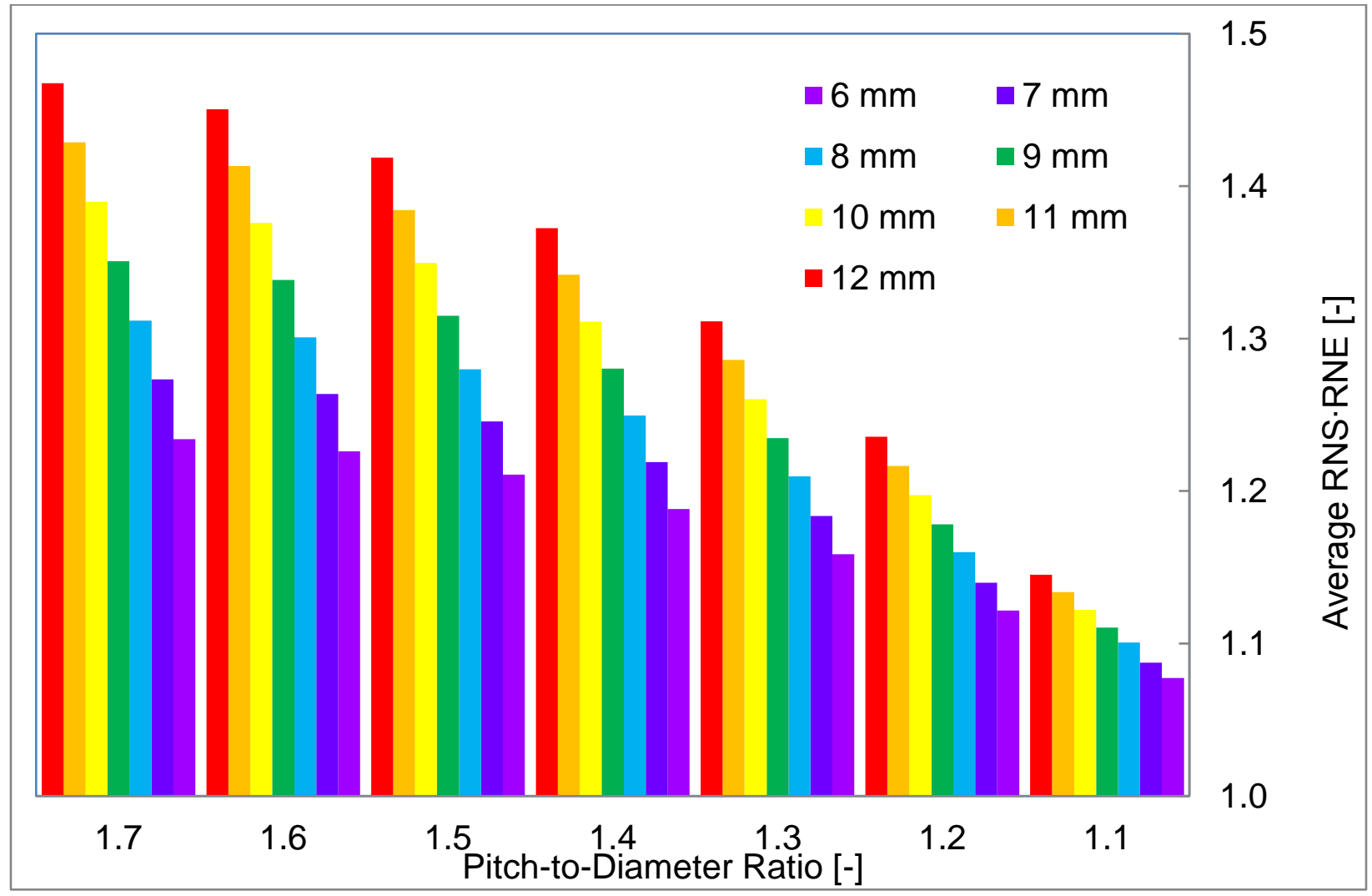

Figure 10 Average heat transfer enhancement in rod bundles due to entrance length effect and spacer grids as a function of the pitch-to-diameter ratio and the pin diameters 
Nevertheless, some issues remain unresolved or have not been addressed in the present paper. One of the fundamental issues of empirical models is that they are knowledge-based. It is only possible to develop them after an experiment. A dedicated experiment has to be performed in order to obtain relationships between the variables of the experiment. Hence, the empirical models are only valid for the boundary conditions (operational parameters, geometry, etc.) under which the experiment has been performed. The applicability of these models will be increased by performing several related studies. Thereby, the empirical models are valid for a wider range of, say, Péclet numbers. Unfavourable is the reduction of accuracy. Imagine one dedicated experiment where the outcome will be a Nusselt number as a function of the Péclet number. For this single experiment a very accurate empirical model can be developed. Increasing the number of considered experiments will lead to a correlation which is valid for all of them in general, but with clear discrepancies to single experiments in particular. That is the case for the presented Nusselt number correlation. In fact, three empirical correlations are used to describe the heat transfer in a rod bundle with spacer grids: the general heat transfer to a bare rod rundle, the multiplier for the entrance length effect and the multiplier for the spacer grid. Due to the multiplication of these three empirical correlations the related uncertainties are also multiplied.

This paper gives only a short overview on the topic of heat transfer enhancement in liquid metalcooled rod bundles. Therefore, some issues have been ignored. These issues include the design of the rod bundle inlet. Depending on the shape of the inlet the heat transfer enhancement will be influenced. Unfortunately, in the literature no information is given on the design. Hence, the design can only be assumed to be of a sudden flow area contraction (from one large plenum into several small rod bundles). Furthermore, the dependency of the spacer grid blockage factor on the heat transfer enhancement was not addressed. It is assumed to be 0.4 but it can be also smaller or larger, depending on the spacer grid design.

These challenges should be elaborated in further study. Nevertheless, the authors think that the presented model for the Nusselt number calculation in a rod bundle with spacer grids and undeveloped flow at the bundle inlet is what comes closest to being best estimate. This study shows that the heat transfer behaviour in a rod bundle with realistic conditions (spacer grids, undeveloped flow) is clearly different form an ideal rod bundle (bare, un-developed flow).

\section{Nomenclature}

$\begin{array}{ll}\mathrm{A} & \text { Constant, } \mathrm{A}=5.55 \\ \mathrm{~B} & \text { Constant, } \mathrm{B}=0.13 \\ d & \text { Diameter } \\ f & \text { Friction factor } \\ h & \text { Heat transfer coefficient } \\ K & \text { Form loss coefficient } \\ l & \text { Length } \\ \mathrm{Nu} & \text { Nusselt number } \\ p & \text { Pitch } \\ \Delta p & \text { Pressure drop } \\ \mathrm{Pe} & \text { Péclet number }\end{array}$




$\begin{array}{ll}q & \text { Heat flux } \\ \text { RNE } & \text { Relative Nusselt number for the entrance region } \\ \text { RNS } & \text { Relative Nusselt number for the spacer grid region } \\ T & \text { Temperature } \\ v & \text { Velocity } \\ x & \text { Length } \\ \varepsilon & \text { Blockage ratio } \\ \rho & \text { Density }\end{array}$

\section{References}

[1] S. Yao, L. Hochreiter and W. Leech, "Heat transfer augmentation in rod bundles near grid spacers", Journal of Heat transfer, Vol. 104, 1982, pp. 76-81.

[2] C.J. Chen and J.S. Chiou, "Laminar and turbulent heat transfer in the pipe entrance region for liquid metals", International Journal on Heat and Mass Transfer Vol. 24, No. 7, 1981, pp. 1179-1189.

[3] U.S. NRC, "TRACE V5.0 Theory manual," US NRC, Washington, D.C., 2012.

[4] P.A. Ushakov, A.V. Zhukov and N.M Matyukhin, "Heat transfer to liquid metals in regular arrays of fuel elements", High Temperature, Vol. 15, 1977, pp. 868-873.

[5] W. Jaeger, W. Hering, N. Diez de los Rios and A. Gonzales, "Validation of TRACE in the field of liquid metal heat transfer", Proceedings of the ASME 2014 International Mechanical Engineering Congress \& Exposition, Montreal, Canada, 2014 November 1420.

[6] J. Marek and K. Rehme, "Experimentelle Untersuchungen der Temperaturverteilung unter Abstandshaltern in glatten und rauhen Stabbündeln (in German)," Gesellschaft fuer Kernforschung mbH, KFK 2128, Karlsruhe, 1975.

[7] K. Rehme, "Pressure drop correlations for fuel element spacers," Nuclear Technology, Vol. 17, 1973, pp. 15-23.

[8] W. Jaeger and V. Sánchez, "Validation of the heat transfer enhancement models for spacer grids in the system code TRACE", Nuclear Engineering and Design, Vol. 265, 2013, pp. 272-287.

[9] W. Jaeger, "Empirical models for liquid metal heat transfer in the entrance region of tubes and rod bundles", Heat and Mass Transfer, doi:10.1007/s00231-016-1929-8, 2016.

[10] M.W. Maresca and O.E. Dwyer, "Heat transfer to mercury flowing in-line through a bundle of circular rods", Journal of Heat Transfer, Transactions of the ASME, Vol. 86, No. 2, 1964, pp. 180-186. 